\title{
Competencias digitales y habilidades de investigación en docentes de secundaria de una institución educativa de Ica, Perú
}

Digital competences and research skills in secondary school teachers from an educational institution in Ica, Peru

\author{
Saul Fredy Torres Cahuana ${ }^{a}$; sel20m@yahoo.es \\ ORCID: https://orcid.org/000-0003-0312-8336 \\ Universidad Católica de Trujillo \\ Gladys Marina Cruzalegui Hoyos ${ }^{\boldsymbol{b}}$ cruzaleguihoyosg@gmail.com \\ ORCID: https://orcid.org/0000-0001-9935-0147 \\ Universidad Católica de Trujillo \\ Janet Ñaña Soldevilla ${ }^{c}$ yeyni1516@gmail.com \\ ORCID: https://orcid.org/0000-0002-9227-1435 \\ Universidad Católica de Trujillo
}

Recibido: Julio / 03 /2021-Revisado: Agosto /15 /2021 - Publicado: Septiembre /30 /2021

\section{RESUMEN}

La investigación abordo como objetivo, determinar la relación entre competencias digitales y habilidades de investigación en los docentes de secundaria de la Institución Educativa Adela Leguía De León del distrito de Santiago, región Ica. Fue un estudio de tipo básico teórico, nivel descriptivo y diseño correlacional transversal, trabajado con una muestra de 60 estudiantes, seleccionado de manera no probabilística. Para la recolección de datos se recurrió a la encuesta electrónica haciendo uso de las escalas de actitud adaptada tipo Likert con validez y confiabilidad. La tabulación de datos y descripción se hizo en el programa Excel para Windows y el análisis de prueba de hipótesis en programa estadístico SPSS versión 25. Las conclusiones de la investigación fueron que, no existe relación directa entre las competencias digitales y las habilidades de investigación en los docentes de secundaria de la Institución Educativa Adela Leguía De León del distrito de Santiago, región Ica 2021, con coeficiente de correlación rho de Spearman $(0,158)$ y nivel de significancia 0,228 $>0,05$. No se corresponde de manera significativa ambas variables; habrá docentes con competencias digitales desarrolladas pero que no lo aplican necesariamente a la investigación.

Palabras claves: Competencias, digitales, habilidades, investigación, relación

\section{ABSTRACT}

The objective of the research on board is to determine the relationship between digital competences and investigative skills in secondary school teachers of the Adela Leguía De León Educational Institution in the district of Santiago, Ica region. It was a study of a theoretical basic type, descriptive level and cross-sectional correlational design, worked with a sample of 60 students, selected in a non-probabilistic way. For data collection, an electronic survey was used, making use of the Likert-type adapted attitude scales with validity and reliability. The tabulation of data and description was done in the Excel program for Windows and the analysis of hypothesis testing in the statistical program SPSS version 25. The conclusions of the research were, there is no direct relationship between digital skills and research skills in teachers from the Adela Leguía De León Educational Institution in the Santiago district, Ica 2021 region, with Spearman's rho correlation coefficient (0.158) and significance level $0.228>0.05$. Both variables do not correspond significantly; there will be teachers with developed digital skills but who do not necessarily apply it to research.

Keywords: Competences, digital, investigative skills, relationship 


\section{Introducción}

La virtualización en la educación es un tema de actualidad por su función en los procesos de enseñanza aprendizaje, la educación semipresencial y los modelos educativos orientados al uso de la tecnología en todos los procesos de gestión, estrategias, recursos y medios para una educación moderna. Canales y Silva (2020 p. 3) considera que "los roles de los actores del sistema educativo en este nuevo escenario cambiaron, adquiere mayor importancia el educando, debiendo los profesores con escasa experiencia en lo no presencial, adaptar recursos, materiales, contenidos y actividades tanto sincrónicas como asincrónicas, para no generar más desigualdades en los aprendizajes".

La evolución tecnológica ha impulsado la transformación digital, es un elemento insustituible en los procesos educativos y a la vez, constituye un derecho fundamental en la educación. La tendencia en una sociedad virtualizada crea necesidades sociales que obliga a los gobiernos del mundo diseñar políticas públicas y la regulación de Estado en materia educativa sobre las modalidades de enseñanza aprendizaje y las nuevas pedagogías en entornos virtuales. No solamente se trata del uso de una plataforma de video clases tipo Meet, Zoom, Jitsi Meet para un dictado de clase, tampoco del uso de plataforma como Moodle, Backboard, Classroom, Elluminate usada como repositorio para subir materiales. Estamos avanzando a un ambiente de aprendizaje regulada por las tecnologías que se expresa en un entorno virtual, donde confluyen las diversas tecnologías asociadas a los contenidos, recursos, materiales, actividades sincrónicas y asincrónicas, los sistemas de seguimiento, retroalimentación y evaluación. (Canales y Silva (2020 p. 3)

La educación como disciplina de las ciencias sociales, encuentra una herramienta apropiada en la virtualidad, no solo para impartir conocimiento en medios de almacenamiento virtual sino mediante la comunicación entre tutor estudiante y entre los mismos estudiantes, mediante las plataformas educativas virtuales. Para Selwyn $(2013$, p. 5) "existe una aceptación generalizada de que las 
tecnologías digitales deben desempeñar un papel integral en la provisión de todos los aspectos del aprendizaje a lo largo de toda la vida, desde la integración de las computadoras en las aulas escolares y universitarias hasta la entrega virtual de cursos en línea y entrenamiento". Las tecnologías digitales pueden potenciar el aprendizaje flexible y abierto asociado con la introducción de los medios sociales en la educación (Salinas, 2013; Marín; Negre; Pérez, 2014).

Sobre las competencias docentes, tomando universalmente, se puede partir desde los enfoques de la revolución industrial de la especialización para cumplir las tareas por los individuos, uso del tiempo adecuado, hasta los procesos administrativos que incluye la planificación, organización, dirección y control establecido en la Teoría General de la Administración. Mas adelante, marcaría las pautas al docente que planifica bien su tiempo, que diseña procesos y momentos claves; que aplica estrategias motivacionales, reflexivas en sus intervenciones con estudiantes, pero que, sobre todo, utiliza recursos variados para cumplir su cometido con éxito. Además, que usa técnicas e instrumentos para evidenciar los resultados de su trabajado en criterios formativos, cognitivos y técnicos (Castells, 2004). En la sociedad del conocimiento del siglo XXI es imperativo que quienes ejercen la docencia estén capacitados en conocimientos, habilidades y actitudes para el uso eficiente e inteligente de las TIC.

Las competencias del docente en la virtualidad se desarrollan y miden en tanto y cuanto sabe usar de la mejor manera los recursos informativos tecnológicos en el ejercicio docente. Para Salinas, De Benito \& Lizana (2014) será necesario que los docentes se desenvuelvan en el mundo digital, en la creación y distribución de contenidos y recursos, en diferentes contextos y con diferentes dispositivos. En este contexto, la docencia en espacios virtuales se presenta como un gran desafío, el docente se formó y aprendió de maestros en la clase presencial, tiene escasa o nula experiencia en la formación virtual y lo que implica ser docente en estos espacios formativos. 
Las dificultades manifiestas en los entornos digitales en la docencia ha sido un espacio crítico para la adaptación en muchos países de Latino América y otros países del mundo con problemas de conectividad y acceso a los recursos tecnológicos. Países como Bolivia, Ecuador Colombia han sufrido los embates de la pandemia en el desarrollo de las clases virtuales. Giraldo (2020 p. 3) del periódico Las 2 Orillas de Colombia, sostiene que "una falencia de gran incidencia en el fracaso de la educación virtual de este país es que la mayoría de los estudiantes en Colombia no tienen un dispositivo tecnológico que les permita interactuar en internet. Este problema es multifactorial. No es un solo culpable el responsable del mismo".

La docencia en países sudamericanos no ha estado a la altura de las necesidades de la educación digitalizada o los entornos virtuales de enseñanza aprendizaje, como muchos países de Europa y el Asia ya lo venía haciendo desde hace variaos años. El mismo autor colombiano replica "que los padres vienen quejándose de los docentes que se la ganan de ojo, sin hacer nada, solo mandando y trabajos por internet mientras que ellos, los padres, son los que se los tienen que aguantar en la casa" (Giraldo 2020)

Por su parte en el Perú la virtualidad ha llegado en las mismas circunstancias que nuestros países vecinos, de sorpresa, sin precedentes tomo la posta de la educación básica regular y educación superior encontrando a los docentes de ambos niveles en condiciones precarias de competencias digitales. Este hecho, no solamente porque los docentes no fueron formados para ello o que jamás se preocuparon por aprender, sino, que los mismos sistemas educativos de gobierno y sus ministerios vinculantes no tenían las políticas implementadas ni los presupuestos suficientes. Lo precedente, ha llevado a la educación a una aparente virtualización dentro de una modalidad de aprendizaje en la que el estudiante tiene que ser más activo y cooperativo en el proceso (Sánchez, 2020; Canaza y Huanca, 2018; Huanca, 2020) así como el docente que está forzado a utilizar herramientas virtuales, aunque le resulte difícil. 
La educación en Perú esta virtualizada hoy, muy a pesar de sus desaciertos y precariedades, los docentes y estudiantes perteneces a una sociedad con otras habilidades y nuevos desafíos en la formación, nuevos valores que obliga a rediseñar los planes de estudio en el ámbito curricular y desarrollar nuevos perfiles en competencias. La sociedad del conocimiento y la información que muy de cerca ha sido trabajado por Castells (2006), Bauman (2003), Wallerstein (1979; 2005; 2007) y Morín (1994), refieren que la sociedad está en un contexto cambiante de carácter digital que exige de las sociedades el manejo y el dominio de las TIC, porque la sociedad flexible de la modernidad líquida que actúa como un sistema se vuelve cada vez más difícil y complejo.

Los docentes del colegio Adela Lengua De Calderón del Caserío de La Venta, distrito de Santiago, provincia de Ica, el $75080 \%$ de docentes no tienen las competencias digitales mínimas que le permitan afrontar con seguridad los desafíos de este contexto de educación remota. Solo con un 20 a $25 \%$ de maestros competentes en habilidades digitales y el resto conoce algo o se puede defender al menos en el manejo de las Tics. La Institución Educativa cuenta con un aula de AIP pero que no abastece a las 21 secciones y 640 estudiantes con las que cuenta la institución. El ancho de banda de la internet es deficiente y no es posible trabajar fluidamente en el aula de innovación con los estudiantes; es más, los docentes no cuentan con acceso a la internet de la institución. Algunos docentes, al inicio de la cuarentena, no contaban incluso con laptop o PC en su casa para el trabajo pedagógico, aparte de que no tenían manejo de esta herramienta tecnológica. El colegio cuenta con un aula de AIP pero que no abastece a las 21 secciones y 640 estudiantes con las que cuenta la institución.

La falta de domino de la virtualidad digital trajo problemas de adaptación e incertidumbre a cómo afrontar las clases de manera virtual. Algunos docentes pidieron licencia por el año y al final pidieron cese al sentirse incapacitados para afrontar ese desafío. La Institución Educativa está situada en una zona de campiña, 
es decir, esta fuera de la ciudad, la población en su mayoría no tiene acceso a internet.

Es de gran necesidad estudiar las competencias digitales, su importancia y relación con otras habilidades de los docentes y estudiantes, como también las habilidades investigativas en el campo de la docencia. Este estudio pretende identificar la relación entre variables, describir las características de cada variable y recomendar acciones de mejora en la ge gestión y el desempeño docente en educación virtual. ¿Cómo se relaciona las competencias digitales y habilidades de investigación en los docentes de secundaria de la Institución Educativa Adela Leguía De León del distrito de Santiago, región Ica 2021?

Estudios realizados como de Chávez, Cantú y Rodríguez (2020) sobre "Competencias digitales y tratamiento de información desde la mirada infantil", publicada en la Revista Electrónica de Investigación Educativa de la Universidad Autónoma de Baja California México, concluyeron qué; los estudiantes se apropiaron de ciertos desempeños del tratamiento de la información y trabaje en equipos en un rango de media alta hacia arriba. Estos desempeños identificados corresponden a la propuesta de competencias digitales de tratamiento de la información en edades más cortas. Los resultados encontrados en esta investigación ponen en contexto que la digitalización en la educación es una herramienta común en los estudiantes de todos los niveles. Segrera, et. al, (2020) publicaron; "Competencias digitales de los futuros profesionales en tiempos de pandemia”. Artículo publicado en la revista Utopía y Praxis Latinoamericana, de la Universidad del Zulia, Venezuela; realizado en 59 estudiantes del décimo semestres, concluyeron que los estudiantes se ubican en un nivel avanzado en dos áreas de conocimiento de competencias digitales. Los niveles son de nivel básico e intermedio en las dimensiones de información y las normas de interacción en ambientes digitales. Estas habilidades son casi naturales en los estudiantes, pues han nacido inmersos en esta era digital y que requieren más bien ser educados en ese contexto. 
Acevedo, et. al, (2020) publicaron sobre "Competencias del docente en educación online en tiempo de COVID-19: Universidades Públicas de Honduras” concluyeron que los docentes mostraron estar a la altura de las necesidades en un $75 \%$ usan bien las plataformas digitales en la Universidad de Honduras, no manifestaron resistencia alguna hacia las herramientas digitales en los procesos seguidos para el aprendizaje, muy a pesar de la crisis. Asimismo, Levano, et. al, (2019) publicaron; "Competencias digitales y educación”, concluyeron que son necesarias cada vez la vertiente de comercio digital como lo dice la OCDE 82021) así como lo son las habilidades en el campo empresarial con respecto al procesamiento de información para hacer efectivas las nuevas tecnologías. Se considera una de las competencias profesionales la gestión de la información digital muy articulada a las competencias digitales.

Estos datos conducen a una sociedad del conocimiento a una sociedad digitalizada porque según infiere, un profesional que no domina o virtualiza sus conocimientos o su forma de impartir conocimiento utilizado la virtualidad, seria dentro de poco o ya lo es un ignorante o iletrado de la época. Seguramente es muy fuerte decirlo en este tono, solo que, al interpretar las investigaciones sobre competencias digitales, no lleva a una reflexión profunda acerca de nuestra praxis como educador frente a una generación con otros talentos en escenarios distintos a la nuestra donde nos formamos.

Rojas, et. al, (2020). Investigaron sobre; "Competencias digitales en una universidad pública peruana”. Da a conocer los hallazgos sobre competencias digitales de los docentes. De los 28 docentes investigados se encontró un nivel bajo del manejo de información con lo que resulta complicado el dominio de crear contenidos. De los 261 estudiantes el dominio de información fue identificado mejor y facilidad en el contenido. Se propuso como desafío potenciar las competencias de los estudiantes de los estudiantes en herramientas tecnológicas y desarrollar las competencias digitales. 


\section{Las competencias digitales}

En la lengua latina, se identifica la forma de "competens" que significa "ser capaz" y en la forma de "competentia", concebida como la capacidad y la permisión. La Real Academia Española (2001, p. 347), competencia, propone una acepción cercana al tema de interés, es "aptitud o idoneidad" mientras que el Diccionario María Moliner sostiene que alguien competente es, además, "quien conoce cierta ciencia o materia, o es experto en la cosa que expresa o a la que se refiere el nombre afectado por competente". Según González y Wagenaar, (2006, p.32) citado por López (2016) nos dice que en el Proyecto Tuning, se indica que las competencias "representan una combinación dinámica de conocimientos, habilidades, capacidades y valores".

Las competencias digitales, para la Comisión Europea identifican ser una competencia importante de las ocho competencias que se conocen para el aprendizaje "el uso seguro y crítico de las tecnologías de la sociedad de la información para el trabajo, el ocio y la comunicación. Se sustenta en las competencias básicas en materia de TIC: el uso de ordenadores para obtener, evaluar, almacenar, producir, presentar e intercambiar información, y comunicarse y participar en redes de colaboración a través de Internet" (Comisión Europea, 2007, p.7).

Según Ferrari (2012), citado por Esteve-Mon y otros (2016) en su estudio presentado por el Joint Research Centre de la Comisión Europea sintetiza múltiples definiciones de esta competencia en los siguientes elementos: (1) Ámbitos de aprendizaje: La competencia digital es el conjunto de conocimientos, habilidades, actitudes, estrategias y valores; (2) Herramientas: Que se requieren cuando se utilizan las TIC y los medios digitales; (3) Áreas: Para realizar tareas, resolver problemas, comunicarse, gestionar información, colaborar, crear y compartir contenidos, y construir conocimiento; (4) Modos: De manera eficaz, eficiente, apropiada, crítica, creativa, autónoma, flexible, ética y reflexiva; y (5) Propósito: Para 
el trabajo, el ocio, la participación, el aprendizaje, la socialización, el consumo y el empoderamiento.

Por su parte, Silva, (2012) refiere que, en 2008, UNESCO planteó los estándares en competencias TIC como marco general al menos tres factores básicos para transformar la educación; alfabetización tecnológica, creación de conocimientos y profundización del mismo, con los componentes del sistema educativo que son seis: política, currículum y plan de estudios, pedagogía, TIC, organización y gestión, y formación y desarrollo de los docentes.

La Unión Europea (2006) citado por Rojas et. al. (2020) define la competencia digital como la función de "recuperar, evaluar, almacenar, producir, presentar e intercambiar información, y para comunicar y participar en redes de colaboración a través de Internet; con el propósito de promover autonomía, eficacia, responsabilidad, crítica reflexiva, al seleccionar, tratar y utilizar la información y sus fuentes; así como las distintas herramientas tecnológicas; y la valoración de la información disponible" (p.16). Tomando al Instituto Nacional de Tecnologías Educativas y de Formación del Profesorado de España (2017), con base a la propuesta de la European Parliament and of the Council (2006), señala que, "la competencia digital, abarca: información, comunicación, creación de contenido, seguridad y resolución de problemas".

\section{Habilidades en investigación}

Partimos de la premisa de Sierra Restituto quien, concibe que toda investigación se origina en dos habilidades naturales del ser humano que es; la curiosidad y la necesidad. El hombre por naturaleza es curioso y tienen la habilidad de recorrer lo desconocido, el encanto por lo novedoso; desde el ámbito filosófico sería la búsqueda de la verdad. Por otro lado, el hombre en su afán de conseguir la felicidad y la calidad de vida, identifica permanentemente necesidades humanas, ya sea en lo biológico, emocional, económico, social, cultural; se convierte en un fiel investigador y creador de conocimientos. 
Sierra (1994: 28), señala que la investigación es "una actividad humana orientada a descubrir algo desconocido". Es decir, el hombre por naturaleza cuenta con la condición innata de la curiosidad que le impulsa a indagar cómo es y por qué es así el mundo que los rodea, entre otras curiosidades que le obliga a investigar para obtener información resolviendo gran parte de sus inquietudes y necesidades.

Considerando el espacio del aula, Stenhouse (1992), define la investigación como la indagación sistemática, mantenida, planificada y autocrítica de la propia práctica. Indagación porque se basa en la curiosidad y en el deseo de comprender un hecho o problema determinado, para el cual puede utilizar las estrategias que aplica como hipótesis de investigación. Según Chirino (2009) las habilidades científico-investigativas son entendidas como "...el dominio de las acciones generalizadoras del método científico que potencian al individuo para la problematización, teorización y comprobación de su realidad profesional, lo que contribuye a su transformación sobre bases científicas."

Las habilidades investigativas se refieren a todas aquellas destrezas que potencian al estudiante para que pueda realizar una investigación de calidad (Moreno, 2005). Son consideradas como un conjunto de acciones que regulan y guían el proceso de investigación (Martínez \& Márquez, 2014) citado por García y otros (2018). Son cinco las habilidades investigativas básicas que se desarrollaron: observar, describir, analizar, sintetizar e interpretar. Por su parte Machado et al. (2008) define la habilidad investigativa como: "El dominio de la acción que se despliega para solucionar tareas investigativas en el ámbito docente, laboral y propiamente investigativo con los recursos de la metodología de la ciencia" (p. 164).

Recurriendo a diferentes fuentes nos encontramos con las clasificaciones más generales de las habilidades investigativas se encuentran: a) habilidades básicas de investigación, habilidades propias de la ciencia particular y habilidades propias de la metodología de la investigación pedagógica (López, 2001); b) habilidades para problematizar, teorizar y comprobar la realidad objetiva (Chirino, 2002); c) 
habilidades de percepción, instrumentales, de pensamiento, de construcción conceptual, de construcción metodológica, de construcción social del conocimiento y metacognitivas (Moreno, 2005); d) habilidades investigativas de mayor integración para la enseñanza del pregrado tales como: solucionar problemas profesionales, modelar, ejecutar, obtener, procesar, comunicar información y controlar (Machado et al., 2008).

Tomando como guía lo de Machado et al., (2008) en el presente estudio, dado las características de la función docente, vincula como elemento básico de las habilidades de investigación basadas en el método científico y sus procesos aplicados a la educción, que son las siguientes dimensiones: problematización de la realidad educativa, besuquea de información actualizada, experimentación de casos nuevos, publicación de resultados de investigación.

\section{Metodología}

La investigación se inició con el proyecto y la recolección de datos se hizo aplicando una escala de actitud en digital a cada docente, que fueron en total 60 profesores del nivel secundaria, cuyos datos fueros tabulados, procesados y analizados usando el programa Excel y SPSS versión 25. Por su finalidad es una investigación básica teórica por qué parte de un marco teórico de la educación en la era digital y habilidades de investigación describiendo y caracterizando cada variable para luego buscar su correlación y un sustento teórico que lo respalde y forme parte del conocimiento científico. Es un estudio descriptivo correlacional de corte transversal de diseño no experimental. 


\section{Resultados}

Tabla $N^{\circ}$ 1:

Competencias digitales, variable y dimensiones

\section{Competencias digitales en docentes de secundaria en la Institución Educativa del Distrito de Santiago, Ica 2021}

\begin{tabular}{rcccccccccc}
\hline Niveles & \multicolumn{1}{c}{ Variable } & \multicolumn{7}{c}{ Dimensiones } \\
\cline { 2 - 11 } & $\begin{array}{c}\text { Competencias } \\
\text { Digitales }\end{array}$ & Información & Comunicación & Creación de & Seguridad \\
& $\boldsymbol{f}$ & $\mathbf{\%}$ & $\boldsymbol{f}$ & $\boldsymbol{\%}$ & $\boldsymbol{F}$ & $\%$ & $\boldsymbol{f}$ & $\mathbf{\%}$ & $\boldsymbol{f}$ & $\boldsymbol{\%}$ \\
Inicio & 0 & $0 \%$ & 5 & $8 \%$ & 4 & $7 \%$ & 4 & $7 \%$ & 3 & $5 \%$ \\
Proceso & 7 & $12 \%$ & 7 & $12 \%$ & 14 & $23 \%$ & 16 & $27 \%$ & 7 & $12 \%$ \\
Lograda & 38 & $63 \%$ & 44 & $73 \%$ & 26 & $43 \%$ & 30 & $50 \%$ & 41 & $68 \%$ \\
Destacada & 15 & $25 \%$ & 4 & $7 \%$ & 16 & $27 \%$ & 10 & $17 \%$ & 9 & $15 \%$ \\
Total & 60 & $100 \%$ & 60 & $100 \%$ & 60 & $100 \%$ & 60 & $100 \%$ & 60 & $100 \%$ \\
\hline
\end{tabular}

Fuente: Base de datos del instrumento

Competencias digitales en docentes de secundaria en la Institución Educativa del Distrito de Santiago, Ica 2021

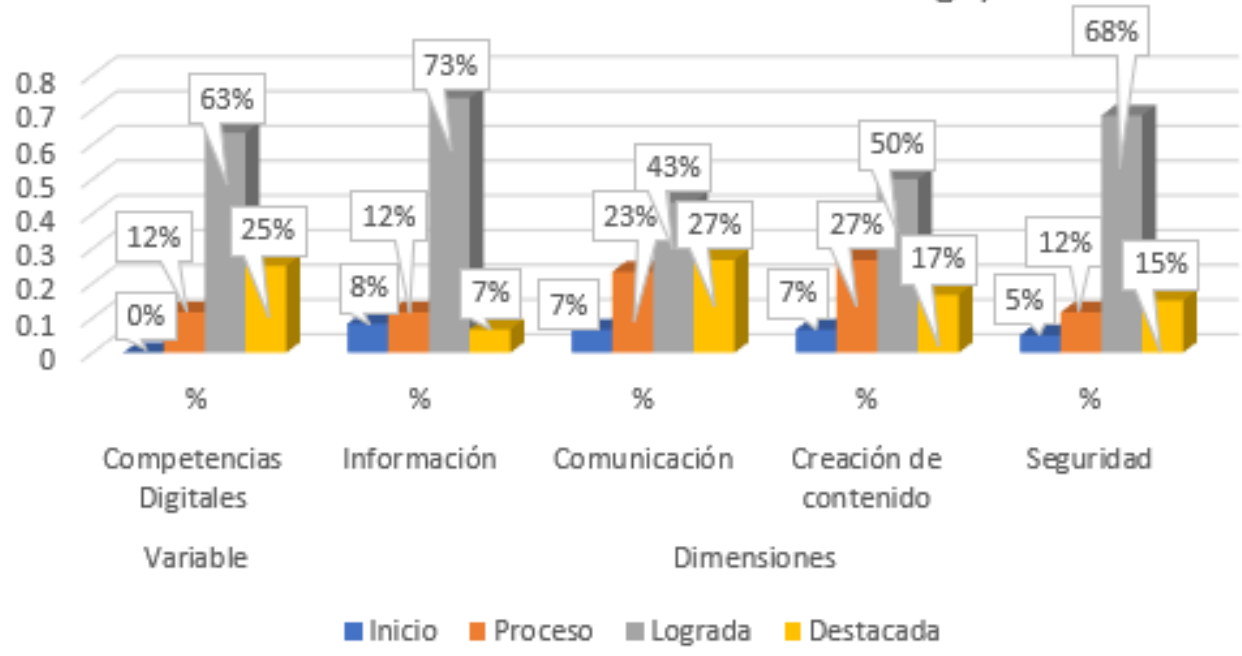

Figura 1: Competencias digitales, variable y dimensiones - Fuente Tabla $\mathrm{N}^{\circ} 1$

En la tabla $\mathrm{N}^{\circ} 1$ y figura $\mathrm{N}^{\circ} 1$ se puede observar que el $63 \%$ de los docentes alcanza un nivel de logrado, con relación a sus capacidades digitales y solo el $12 \%$ se encuentra en el nivel de progreso. Ahondando en esto, notamos que el $73 \%$ de la población tiene un nivel de logrado sobre a la dimensión de información y solo el $8 \%$ se encuentra en un nivel de inicio; en la dimensión de comunicación encontramos que el $43 \%$ se encuentra en un nivel de logrado y solo el $7 \%$ se 
encuentra en el nivel de inicio; mientras que en la dimensión creación de contenido, el $50 \%$ de los profesores tiene un nivel de logrado y $7 \%$ un nivel de inicio y, finalmente la capacidad de seguridad, el 68\% tiene un nivel de logrado y solo el $5 \%$ un nivel de inicio. Según estos datos, los docentes han desarrollado esta competencia, no hay destacados en manejo digitales, pero si hay, aunque bajas proporciones docentes que aún no han desarrollado la competencia, similar caso para las dimensiones.

Tabla $N^{\circ}$ 2:

Habilidades de investigación, variable y dimensiones

\section{Habilidades de investigación en docentes de secundaria en la Institución Educativa del Distrito de Santiago, Ica 2021}

\begin{tabular}{|c|c|c|c|c|c|c|c|c|c|c|}
\hline \multirow{4}{*}{ Niveles } & \multicolumn{2}{|c|}{ Variable } & \multicolumn{8}{|c|}{ Dimensiones } \\
\hline & \multirow{2}{*}{\multicolumn{2}{|c|}{$\begin{array}{l}\text { Habilidades } \\
\text { de } \\
\text { investigación }\end{array}$}} & \multicolumn{8}{|c|}{ Problematizació Búsqueda de Experimentació Publicacione } \\
\hline & & & & $n$ & $\operatorname{Info}$ & mación & & $n$ & & $s$ \\
\hline & $f$ & $\%$ & $f$ & $\%$ & $\boldsymbol{F}$ & $\%$ & $f$ & $\%$ & $f$ & $\%$ \\
\hline Inicio & 0 & $0 \%$ & 2 & $3 \%$ & 3 & $5 \%$ & 2 & $3 \%$ & 2 & $3 \%$ \\
\hline Proceso & 2 & $3 \%$ & 0 & $0 \%$ & 9 & $15 \%$ & 1 & $2 \%$ & 5 & $8 \%$ \\
\hline Lograda & 16 & $27 \%$ & 32 & $53 \%$ & 37 & $62 \%$ & 25 & $42 \%$ & 28 & $47 \%$ \\
\hline Destacada & 42 & $70 \%$ & 26 & $43 \%$ & 11 & $18 \%$ & 32 & $53 \%$ & 25 & $42 \%$ \\
\hline Total & 60 & $100 \%$ & 60 & $100 \%$ & 60 & $100 \%$ & 60 & $100 \%$ & 60 & $100 \%$ \\
\hline
\end{tabular}

Fuente: Base de datos del instrumento

\section{Habilidades de investigación en docentes de secundaria en la Institución Educativa del Distrito de Santiago, Ica 2021}

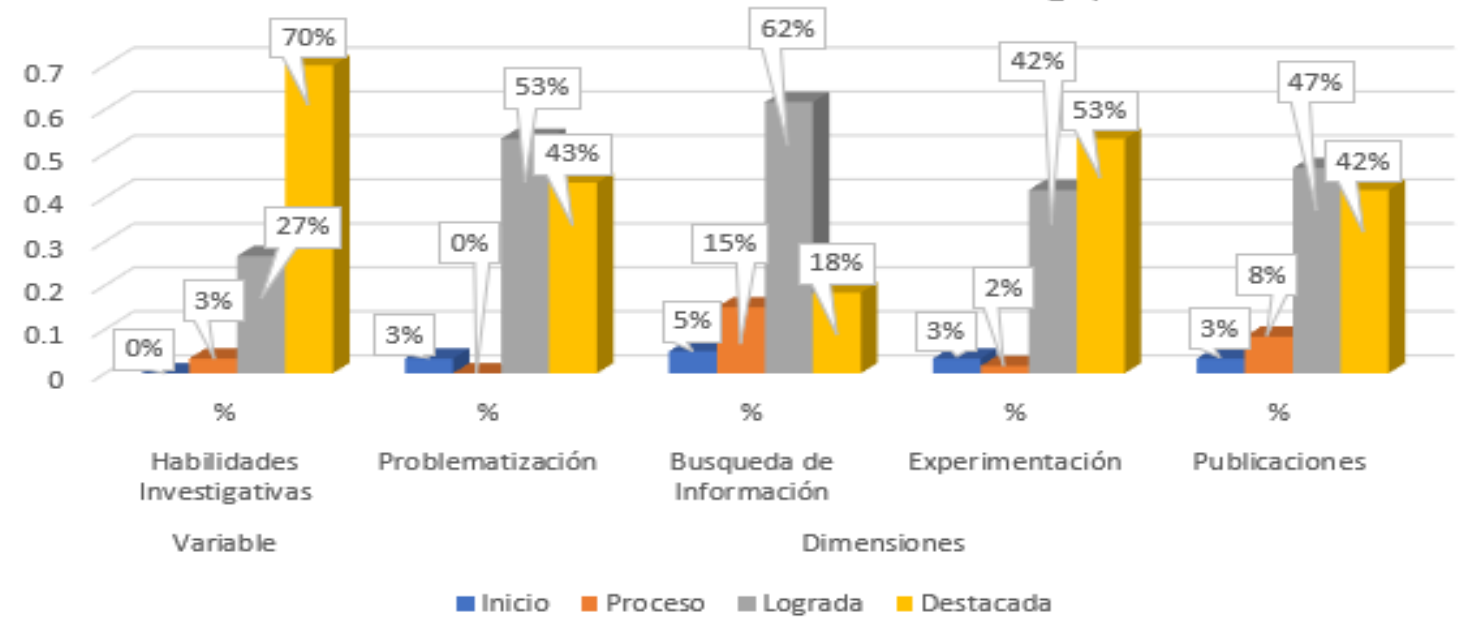

Figura 2: Habilidades investigativas, variable y dimensiones - Fuente tabla $\mathrm{N}^{\circ} 2$ 
En la tabla $\mathrm{N}^{\circ} 2$ y figura $\mathrm{N}^{\circ} 2$ encontramos que el $70 \%$ de los docentes posee un destacado nivel y solo el 3\% se encuentra en un nivel de progreso con relación a las habilidades investigativas. Profundizando en las dimensiones notamos que el $53 \%$ de los docentes tiene un nivel de logrado en relación a la problematización y solo el $3 \%$ se encuentra en un nivel de inicio; en este misma línea el $62 \%$ de los mismos tiene un nivel de logro en relación a la búsqueda de información y solo el $5 \%$ presenta un nivel de inicio; por su parte con la habilidad de experimentación el $53 \%$ de los docentes señala tener un nivel de destacado y solo el $3 \%$ reconoce estar en el nivel de inicio mientras que el $2 \%$ en el nivel de progreso y por último, en la habilidad de publicación el $47 \%$ de la población se encuentra en el nivel de logro y solo el $3 \%$ está en el nivel de inicio.

Los resultados evidencian un buen nivel de los docentes en investigación, eso quiere decir que desarrollan estas habilidades en la práctica con sus alumnos. La dimensión que destaca es experimentación con $53 \%$ de sus docentes que hace experimentos sencillos con sus estudiantes. Esto confirmaría lo que, Torres (1999:25) señala que la investigación en el aula promovida por el docente "estimula en los alumnos la curiosidad, la necesidad de saber, de preguntar, de explorar, de comprobar, de experimentar, de perfeccionar, de aprender por deseos, no por miedo ni por obligación".

Tabla N³: Correlación de las variables competencias digitales y habilidades de investigación

\section{Correlaciones}

\begin{tabular}{|c|c|c|c|c|}
\hline \multirow{3}{*}{$\begin{array}{l} \\
\text { Rho de } \\
\text { Spearman }\end{array}$} & & \multicolumn{2}{|c|}{ TV1 } & \multirow{2}{*}{$\frac{\text { TV2 }}{, 158}$} \\
\hline & \multirow{3}{*}{$\begin{array}{l}\text { V1: } \\
\text { Competencias } \\
\text { digitales }\end{array}$} & Coeficiente de correlación & 1,000 & \\
\hline & & Sig. (bilateral) & & ,228 \\
\hline & & $\mathrm{N}$ & 60 & 60 \\
\hline & $\begin{array}{l}\text { V2: Habilidades } \\
\text { de investigación }\end{array}$ & Coeficiente de correlación & ,158 & 1,000 \\
\hline & & Sig. (bilateral) &, 228 & \\
\hline & & $\mathrm{N}$ & 60 & 60 \\
\hline
\end{tabular}




\section{Coeficiente de Correlación "rho" de Spearman = 0,158}

Ubicando el resultado se tiene que rho $=0,158$, se encuentra entre el rango de $(0,10$ y 0,24$)$ lo que se ubica en la correlación considerado como correlación positiva muy débil entre las competencias digitales y las habilidades de investigación en los docentes de secundaria en la Institución Educativa del Distrito de Santiago, Ica 2021.

La decisión estadística permite concluir, puesto que t calculada es menor que "t" teórica $(1.22<1,96), r(0,158)>0.10$; sig. 0,228 > 0,05 en consecuencia, se rechaza la hipótesis alterna $(\mathrm{Hi})$ y se acepta la hipótesis nula $(\mathrm{Ho})$. Se concluye que no existe relación entre las competencias digitales y las habilidades de investigación en los docentes de secundaria en la Institución Educativa del Distrito de Santiago, Ica 2021. Es decir, $x=$ tc $(1.22)<t(1,96)$

\section{Discusión}

Considerando a Ferrari (2012), citado por Esteve-Mon y otros (2016) sintetiza definiciones de competencias digitales: (1) Ámbitos de aprendizaje: La competencia digital es el conjunto de conocimientos, habilidades, actitudes, estrategias y valores; (2) Herramientas: Que se requieren cuando se utilizan las TIC y los medios digitales; (3) Áreas: Para realizar tareas, resolver problemas, comunicarse, gestionar información, colaborar, crear y compartir contenidos, y construir conocimiento; (4) Modos: De manera eficaz, eficiente, apropiada, crítica, creativa, autónoma, flexible, ética y reflexiva; y (5) Propósito: Para el trabajo, el ocio, la participación, el aprendizaje, la socialización, el consumo y el empoderamiento.

Se puede afirmar que las competencias digitales desarrollan otras habilidades personales más que preparan operativamente y de manera cognitiva a las personas en contacto con el mundo digital, pero que no necesariamente son para hacer ciencia o investigar propiamente, aun cuando la búsqueda de información ya es parte de la investigación. Sucede que, no todo experto en el uso 
de las TIC o herramientas digitales es investigador. Las habilidades para investigar no dependen de un buen uso de la digitalización, esta facilita de manera sustancial sí, pero la investigación puede hacerse sabiendo poco o algo de digitalización, no siendo condicionante.

Se entiende por habilidades investigativas según Chirino (2009) las habilidades científico-investigativas son entendidas como "...el dominio de las acciones generalizadoras del método científico que potencian al individuo para la problematización, teorización y comprobación de su realidad profesional, lo que contribuye a su transformación sobre bases científicas".

La problematización, teorización y comprobación, como lo presenta el autor son habilidades de la persona que lo caracteriza por la forma como aborda los problemas de una teoría, encuentra vacíos en ella y recoge información para analizarlos y llegar a conclusiones. Estas habilidades, requieren de muchos otros factores que contribuyen a desarrollar o descubrir nuevo conocimiento, sin embargo, ¿está condicionado al uso de la tecnología? ¿puede un investigador ser experto en digitalización moderna? Ambas cosas si son parte del perfeccionamiento de acciones investigativas, pero no es determinante.

Como lo confirma el Consejo de Administración del FONDEP (2019 p. 7) "Para investigar no debemos realizar grandes cambios, solo necesitamos tener las ganas de practicar una nueva forma de docencia". Para la investigación el docente puede hacer uso de su propios recursos y habilidades, adaptar las herramientas que más se apresten a sus necesidades. Una de esas herramientas es el uso de los medios tecnológicos, la digitalización, pero aún hay docentes que leen em bibliotecas físicas, que hacen fichaje manual y elaboran separatas impresas, no está mal porque es su habilidad investigativa mientras aprendan con calma el suso tecnológico.

Se puede decir que las habilidades investigativas en los docentes de Educación Básica Regular, van desarrollando. El Minedu hizo una serie de talleres de aprendizaje 
realizados por el FONDEP, durante 2017, con docentes de Lima y Cajamarca, mostró que, pese a estas dificultades, los docentes contaban con experiencias valiosas y mucho interés para promover cambios e implementar proceso de investigación-acción participativa en educación.

\section{Conclusiones}

- No existe relación directa entre las competencias digitales y las habilidades de investigación en los docentes de secundaria de la Institución Educativa Adela Leguía De León del distrito de Santiago, región Ica 2021, con coeficiente de correlación rho de Spearman $(0,158)$ y nivel de significancia $0,228>0,05$. No se corresponde de manera significativa ambas variables; habrá docentes con competencias digitales desarrolladas pero que no lo aplican a la investigación. No hay relación entre la variable competencias digitales y las dimensiones de la variable habilidades de investigación (0.123 ) nivel de significancia $0,350>0,05$. (0.232) nivel de significancia 0,075 $>0,05$. (0.104) nivel de significancia de 0,428 >0,05. (0.210) nivel de significancia 0,107 >0,05.

- La investigación como actividad docente es inherente a la pedagogía y la investigación formativa, sin embargo, no depende de las competencias digitales para su efectividad, consideramos que le haría muy bien a los docentes ayudarse con las herramientas tecnológicas para la busque da de información, para la publicación y difusión de ensayos y artículos en base de datos digitales, ya sea del trabajo de los docentes mismo o la producción d ellos estudiantes.

\section{Referencias}

Acevedo Á.; Argüello A; Pineda B. G y Urcios, P.W. (2020) Competencias del docente en educación online en tiempo de COVID-19: Universidades Públicas de Honduras. Revista de Ciencias Sociales (Ve), vol. 26. Universidad del Zulia, Venezuela. https://www.redalyc.org/articulo.oa?id=28064146014. 
Canales R. Silva J. (2020) De lo presencial a lo virtual, un modelo para el uso de la formación en línea en tiempos de Covid-19. DOSSIER - Cultura digital y educación. Educ. rev. 36 2020. https://doi.org/10.1590/0104-4060.76140

Canaza A. \& Huanca J. W. (2018). Hacia una Educación Intercultural Bilingüe sentipensante. Sciendo, 21(4), 515-522. https://doi.org/10.17268/sciendo.2018.058

Castells, M. (2006). La sociedad red: Una visión Global. Alianza Editorial.

Consejo de Administración del FONDEP (2019) La escuela que investiga: Una herramienta para implementar procesos de investigación-acción participativa en educación. Ministerio de Educación. https://www.fondep.gob.pe/wpcontent/uploads/2020/01/LAESCUELA_QUE_INVESTIGA.pdf

Chávez F. Cantú M. Rodríguez C. M. María (2020) Competencias digitales y tratamiento de información desde la mirada infantil. Revista Electrónica de Investigación Educativa E-ISSN: 1607-4041. Universidad Autónoma de Baja California México. Disponible en: http://www.redalyc.org/articulo.oa?id=15543298015.

Chirino M. V. (2009) Perfeccionamiento de la formación inicial investigativa de los profesionales de la educación. Tesis presentada en opción al Grado Científico de Doctor en Ciencias Pedagógicas. http://scielo.sld.cu/scielo.php?script=sci_arttext\&pid=S1727-81202009000100003

España. Ministerio de Educación y Ciencia. (2006). Legislación Consolidada. Boletín Oficial del Estado. http:// www.uco.es/organizacion/secretariageneral/images/ doc/docs/legislacion/BOE_Ley_Educacion.pdf

Esteve-Mon, Francesc M.; Gisbert-Cervera, Mercè; Lázaro-Cantabrana, José Luis (2016) La competencia digital de los futuros docentes: ¿cómo se ven los actuales estudiantes de educación? Perspectiva Educacional, Formación de Profesores, vol. 55, núm. 2, junio-, 2016, pp. 38- 54 Pontificia Universidad Católica de Valparaíso Viña del Mar, Chile.

Ferrari, A. (2012). Digital competence in practice: An analysis of frameworks. Sevilla: European Commission, Joint Research Centre (JRC).

Giraldo A. F. (2020) El fracaso de la educación virtual en Colombia. Diario Las dos Orillas. Colombia. $\quad$ https://www.las2orillas.co/el-fracaso-de-la-educacion-virtual-encolombia/

Huanca J. W. (2020). Contrahegemonía y la lucha por la educación en el sur del Perú. Editorial Académica Española.

INTEPE (2017) Instituto Nacional de Tecnologías Educativas y de Formación del Profesorado de España (2017),

Levano L; Sánchez S; Guillén P; Tello S; Herrera N. \& Collantes Z. (2019). Competencias digitales y educación. Propósitos y Representaciones, 7(2), 569588. https://dx.doi.org/10.20511/pyr2019.v7n2.329 
López E. (2016) En torno al concepto de competencia: un análisis de fuentes. Revista del currículo y formación del profesorado, VOL. 20, $\mathrm{N}^{\mathrm{o}} 1$ (enero-abril) https://www.redalyc.org/pdf/567/56745576016.pdf

López Balboa, L. (2001). El desarrollo de las habilidades de investigación en la formación inicial del profesorado de química. (Tésis inédita de doctorado). Universidad de Cienfuegos Carlos Rafael Rodríguez, Cienfuegos, Cuba.

Machado Ramírez, E. F., Montes de Oca Recio, N., \& Mena Campos, A. (2008). El desarrollo de habilidades investigativas como objetivo educativo en las condiciones de la universalización de la educación superior. Pedagogía Universitaria. XIII (1), 156-180. Recuperado de http://revistas.mes.edu.cu/PedagogiaUniversitaria/articulos/2008/numero/189408108. pdf

Marín, V. Negre, F. Pérez A. (2020) Entornos y redes personales de aprendizaje (plepln) para el aprendizaje colaborativo [Construction of the Foundations of the ple and pln for Collaborative learning]. Comunicar, España, v. 21, n. 42, p. 35-43, 2014. Disponible en: https://doi.org/10.3916/C42-2014-03. Acceso en: 23 abr. 2020

Martínez, D., \& Márquez, D. (2014). Las Habilidades Investigativas como Eje Transversal de la Formación para la Investigación. Tendencias Pedagógicas, 347-360.

(Martínez \& Márquez, 2014)

Moreno, M.G. (2005) Potenciar la educación. Un currículum transversal de formación para la investigación. Revista Electrónica Iberoamericana sobre Calidad, Eficacia y Cambio en Educación, 3 (1), 520-540. Recuperado de http://dialnet.unirioja.es/servlet/articulo?codigo=1130331

Real Academia Española (2001). Diccionario de la Lengua Española. Tomo I. Madrid: Espasa-Calpe. Voces: competencia, competente.

Rojas V.R; Zeta A; \& Jiménez R. (2020). Competencias digitales en una universidad pública peruana. Conrado, 16(77), 125-130. Epub 02 de diciembre de 2020. Recuperado en 29 de junio de 2021, de http://scielo.sld.cu/scielo.php?script=sci_arttext\&pid=S1990$\underline{86442020000600125 \& \operatorname{lng}=e s \& t \operatorname{lng}=e s}$.

Salinas, J. (2014) Enseñanza flexible y aprendizaje abierto, fundamentos clave de los ples. En: LINDA Castañeda; Jordi Adell (ed.). Entornos personales de aprendizaje: Claves para el ecosistema educativo en Red. Alcoy: Marfil, 2013. p. 53-70.

Sánchez, J. A. (2020, March 24). Virtualidad: la pandemia que cambió la educación superior para siempre. El Tiempo, 1-6. https://www.eltiempo.com/vida/ciencia/educacion-virtual-la-pandemia-que-cambiola-educacion-superior-para-siempre-476390

Segrera J. R., Pae H. D. y Polo A. A. (2020) Competencias digitales de los futuros profesionales en tiempos de pandemia. Utopía y Praxis Latinoamericana, vol. 25, 
núm. Esp.11. Universidad del Zulia, Venezuela. DOI: https://doi.org/10.5281/zenodo.4278352.

Silva, J. (2012). Estándares tic para la formación inicial docente: Una política pública en el contexto chileno. Archivos Analíticos de Políticas Educativas, 20(7), 1-36

Sierra B., R. (1994) Técnicas de investigación social. Teoría y ejercicios. $9^{a}$ ed. Madrid: Paraninfo.

Stenhouse, L. (1991). Investigación y desarrollo del currículo (3a. ed.) Madrid: Morata.

Torres, R. (1999). El maestro investigador, la investigación en el aula. Barcelona: Grao.

Unión Europea. (2006) Recomendación del Parlamento Europeo y del Consejo sobre las competencias clave para el aprendizaje permanente. https://eur-lex.europa.eu/LexUriServ/LexUriServ.do?uri=OJ:L:2006:394:0 010:0018:ES:PDF

\section{@ $\odot \Theta \Theta$}

Competencias digitales y habilidades de investigación en docentes de secundaria de una institución educativa de Ica, Perú (Saul Fredy Torres - Cahuana) Por Revista Sendas se encuentra bajo una Licencia Creative Commons-No Comercial-Sin Derivadas 3.0 Uported. 